\title{
Rotational Diffusion of Spherical Colloids Close to a Wall
}

\author{
S. A. Rogers, ${ }^{1}$ M. Lisicki, ${ }^{2}$ B. Cichocki, ${ }^{2}$ J. K. G. Dhont, ${ }^{1}$ and P. R. Lang ${ }^{1, *}$ \\ ${ }^{1}$ ICS-3, Institute of Complex Systems, Forschungszentrum Jülich, D-52425 Jülich, Germany \\ ${ }^{2}$ Institute of Theoretical Physics, Faculty of Physics, University of Warsaw, ul. Hoża 69, 00-681 Warsaw, Poland
}

(Received 3 May 2012; published 30 August 2012)

\begin{abstract}
There is currently no experimental technique available to probe spatially resolved rotational diffusion of nanoparticles in the vicinity of a wall. We present the first experimental study of rotational diffusion of small spherical colloids, using dynamic evanescent wave scattering. A setup is used where the wave vector components parallel and perpendicular to the wall can be varied independently, and an expression is derived for the first cumulant of the intensity correlation function in $\mathrm{VH}$ evanescent wave geometry for optically anisotropic spheres. The experimental results are in agreement with theoretical predictions that take particle-wall hydrodynamic interactions into account.
\end{abstract}

DOI: 10.1103/PhysRevLett.109.098305

PACS numbers: 47.57.J-, 45.20.dc, 47.85.Dh

Rotational diffusion is an important feature in the study of many physical, chemical, and biological topics. The range of topics include gaseous combustion, where rotational diffusion is essential for the interpretation of coherent anti-Stokes Raman spectra [1]; microrheology, where frequency-dependent viscoelastic shear moduli can be measured through rotational diffusion of a tracer sphere [2]; rotational motion of biomacromolecules in membranes (like rhodopsin chromophores [3] and proteins in the human erythrocyte membrane [4]); and rotational-diffusion controlled chemical reactivity [5-7]. For macromolecular systems, there has been an abundance of literature over the last decade on rotational diffusion in bulk. Very much less is known about rotational diffusion in confining geometries for such systems. The effect of confinement on the motion of macromolecules in solution is of essential importance, for example, in the reduced-scale world of micro- [8], nano- [9], and optofluidics [10]; in possible microfluidics applications, where a single colloidal particle can be used to act as a pump or valve [11]; and in "labs-on-a-chip" applications, like capillary electrophoresis [12] and sorting of white blood cells [13]. This effect dominates when the dimensions of the confining geometry and the suspended particles are comparable.

The hindrance by confining geometry is largely determined by the hydrodynamic interactions of macromolecules with the walls $[14,15]$. Moving through the solvent, a macromolecule creates a fluid flow that is reflected by the walls, affecting its motion. Diffusion coefficients of colloids are drastically reduced on the approach of a wall, and attain their bulk values for distances a few particle diameters away from the wall. Hydrodynamic interactions are thus essential to understand the diffusive motion of macromolecules in confining geometries.

Despite its possible importance in biology and technological applications, very little experimental work has been done on the rotational motion of macromolecules near a wall. The few papers in this area employ light microscopy to image single particles. Rotational diffusion of micrometersized ellipsoids can be monitored through their orientation, where it is found that in quasi two-dimensional confinement, the anisotropy of diffusion is strongly affected [16]. Two-dimensional rotational diffusion of micron-sized rods consisting of DNA-linked spherical colloids has been probed by direct imaging [17]. The measurement of rotational diffusion of spheres is much more complicated. Lobo et al. [18] probed the rotational diffusion coefficient of a micron-sized magnetic bead by attaching the sphere to a short platinum nanorod that is in turn attached to a DNA molecule. From the fluctuations of the twist angle of the DNA, the rotational diffusion coefficient for rotation along the axis perpendicular to the wall can be calculated. In an unpublished work [19], rotational diffusion of large fluorescent spherical colloids has been measured by bleaching a stripe over the surface of the spheres, and imaging the orientation of the stripe as a function of time.

In this Letter, we present an alternative experimental technique to probe rotational diffusion of spheres, relying on evanescent wave scattering. In order to interpret intensity correlation functions, an expression is derived for their initial decay rates in terms of local rotational diffusion coefficients and the polarization state of the detected light. Experiments are performed on optically anisotropic polytetrafluoroethylene (PTFE) spheres (Ausimont) suspended in a saline aqueous solution. The salinity of the solvent is sufficient to ensure hard-core interactions between the colloids and the wall. The radius of the spheres was determined by bulk depolarized dynamic light scattering (DDLS) measurements to be $a=110 \mathrm{~nm}$.

Evanescent-wave dynamic light scattering (EWDLS) [20] is an ideal tool for studying the dynamics of suspended colloids close to smooth, impenetrable, solid walls. The evanescent wave's exponentially decaying field ensures that only particles within a critical distance from the wall are probed. The distance $2 / \kappa$ over which the field $\mathbf{E}$ decays by a factor $1 / e$, the penetration depth [21], has an experimental 
lower limit of $\sim 100 \mathrm{~nm}$. Previous EWDLS studies of suspended colloids [21,22] have been limited to measurements of averaged apparent translational diffusion coefficients. Using EWDLS, Loppinet et al. [23] measured a difference in collective orientation rates of hairy-rod-type polymers adsorbed on a surface, compared with bulk measurements of semidilute solutions. Here we present a method that enables the probing of translational and rotational (both parallel and perpendicular to the interface) diffusion of colloids close to walls. Our experiments are the first to assess the possibilities for EWDLS as a method to probe near-wall rotational dynamics, and constitute the first full experimental verification of the particle-wall hydrodynamical interaction theory $[14,15]$.

Experimentally, EWDLS is similar to DDLS, the only difference being the method of illumination; in DDLS, a laser impinges directly upon the sample, while in EWDLS the laser's function is in the launching of an evanescent wave into the region of lower refractive index under total internal reflection conditions. The convention in DDLS measurements is for the incident field to be polarized in the vertical direction and the scattered field to be observed in the horizontal plane [24]. Scattered photons are observed after passing through a polarizer that allows vertical or horizontal polarizations. These conventions give rise to the terms "VV" and "VH" (vertical-vertical and verticalhorizontal), which denote the relative polarizations prior to and after scattering. In VV geometry, in both bulk experiments and tests in the presence of a wall, fluctuations in the field intensity are caused mainly by translational motion. The contribution from rotational diffusion (see Supplemental Material [25]) is proportional to the square of the complex polarizability anisotropy $\Delta \epsilon$, which is normally small. Fluctuations in the field in VH conditions contain information about both translational and rotational motion [24] without any anisotropy dependence. To keep with the convention of DDLS, we polarize the incident laser in our setup [21] (Fig. 1) horizontally and denote this as "vertical" polarization. The incident illumination is

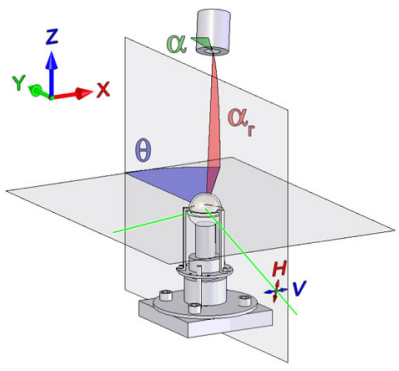

FIG. 1 (color online). Experimental geometry. Laser light is totally internally reflected at the planar surface of a glass hemisphere, setting up an evanescent wave at the glass-fluid interface. Scattered photons pass through an angled polarizer on the detector, which can be placed anywhere on the surface of a solid quadrant. The "horizontal" and "vertical" polarizations are indicated. therefore denoted as $\hat{\mathbf{n}}_{0}=(1,0,0)$. With a polarizer on our detecting unit, angled at an amount $\alpha$, the scattered photons in a $\mathrm{VH}$ configuration are denoted $\hat{\mathbf{n}}_{S}=$ $\left(0, n_{S, y}, n_{S, z}\right)$. Our unique setup allows for the observation of scattered photons in a full solid quadrant.

In an EWDLS experiment, the quantity of interest that contains dynamic information is the time-autocorrelation function of the scattered field, $g_{1}(\mathbf{Q}, t)$, defined by

$$
g_{1}(\mathbf{Q}, t)=\frac{\left\langle\mathbf{E}_{S}(\mathbf{Q}, t=0) \cdot \mathbf{E}_{S}^{\star}(\mathbf{Q}, t)\right\rangle}{\left\langle\left|\mathbf{E}_{S}(\mathbf{Q}, t=0)\right|^{2}\right\rangle},
$$

where $\mathbf{E}_{S}$ is the scattered field, $\mathbf{Q}$ the scattering vector [21], and $t$ the time. The scattered field is proportional to

$$
\begin{aligned}
E_{S}(\mathbf{Q}, t) \propto & \left\{\hat{\mathbf{n}}_{S} \cdot \breve{\varepsilon}[\hat{\mathbf{u}}(t)] \cdot \hat{\mathbf{n}}_{0}\right\} \exp [-i \mathbf{Q} \cdot \mathbf{r}(t)] \\
& \times \exp [-\kappa z(t) / 2],
\end{aligned}
$$

where $\hat{\mathbf{n}}_{S}$ is the director of the polarized scattered field, $\hat{\mathbf{n}}_{S} \cdot \breve{\varepsilon}[\hat{\mathbf{u}}(t)] \cdot \hat{\mathbf{n}}_{0}$ is the oriented dielectric tensor, $\mathbf{r}(t)$ is the position of a scatterer, and $\exp [-\kappa z(t) / 2]$ describes the decay of the evanescent wave in the $z$ direction, where $\kappa$ is the penetration depth of the evanescent wave.

As the analytic expression for the full autocorrelation function is not known, we use the short-time behavior to elucidate the particle dynamics. The first cumulant, $\Gamma$, is determined experimentally by

$$
\Gamma=\lim _{t \rightarrow 0}-\frac{\partial g_{1}(\mathbf{Q}, t)}{\partial t} .
$$

From VV experiments, we determine (see Supplemental Material [25]) the quantity,

$$
\Gamma^{t}=\left\langle D_{\|}^{t}\right\rangle_{\kappa} Q_{\|}^{2}+\left\langle D_{\perp}^{t}\right\rangle_{\kappa}\left(Q_{\perp}^{2}+\frac{\kappa^{2}}{4}\right),
$$

which is the contribution to the initial decay of $g_{1}(\mathbf{Q}, t)$ from translational diffusion, and for which the $\kappa$ average $\langle\ldots\rangle_{\kappa}$ is defined below. The rotational diffusion contribution to $\Gamma^{\mathrm{VV}}$ is proportional to the square of the complex polarizability anisotropy $\Delta \epsilon$, which is estimated to alter $\Gamma^{\mathrm{VV}}$ by roughly $1 \%$. We therefore neglect this contribution so that $\Gamma^{\mathrm{VV}}=\Gamma^{t}$. In Eq. (4), $\left\langle D_{\|}^{t}\right\rangle_{\kappa}$ and $\left\langle D_{\perp}^{t}\right\rangle_{\kappa}$ are the " $\kappa$-average" translational diffusion constants parallel and perpendicular to the interface. The $\kappa$ average $\langle D\rangle_{\kappa}$ of a diffusion constant [26] is

$$
\langle D\rangle_{\kappa}=\kappa \int_{z>a} d z \exp [-\kappa(z-a)] D(z),
$$

where $a$ is the radius of the spheres. Similarly, $Q_{\|}$and $Q_{\perp}$ are the components of the scattering vector parallel and perpendicular to the wall, over which we have independent control [21]. In the VH configuration, we measure initial decays of $g_{1}(\mathbf{Q}, t)$ that are described by

$$
\Gamma^{\mathrm{VH}}=\Gamma^{t}+\left\langle D_{\|}^{r}\right\rangle_{\kappa}\left(2+3 n_{S, z}^{2}\right)+\left\langle D_{\perp}^{r}\right\rangle_{\kappa}\left(1+3 n_{S, y}^{2}\right)
$$

(see Supplemental Material [25]), where $\Gamma^{t}$ is defined in Eq. (4). As in conventional DDLS measurements, the VH 
configuration provides a way of decoupling translational and rotational diffusive dynamics. Note that decay rates depend on the particle size only through the combination $\kappa a$. Equation (6) is an exact result. The first cumulant in the VH geometry does not depend on the optical anisotropy of the particles. However, the influence of polarizability anisotropy is essential, as the electric field autocorrelation function is proportional to the anisotropy modulus squared (see Supplemental Material [25]).

An examination of Eqs. (4) and (6) reveals that the rotational diffusive contribution to the initial decay of $g_{1}(\mathbf{Q}, t)$ depends on both $Q^{2}=Q_{\perp}^{2}+Q_{\|}^{2}$ and the spatial components of the detector polarization, while the translational diffusive contribution depends on $Q^{2}$ only. With our setup, we can ensure that VH conditions are strictly kept while scanning a range of scattering vectors. This is achieved by fixing the detector polarizer, while simultaneously altering $\theta$ and $\alpha_{r}$ (see Fig. 1). Because $\theta$ and $\alpha_{r}$ change throughout the experiment while $\alpha$ does not, we obtain a data set with different spatial components $n_{S, y}$ and $n_{S, z}$ over a range of scattering vectors.

We show, as the filled symbols in Fig. 2, the results of EWDLS scans for penetration depths of $(\kappa a)^{-1}=0.6$ and $(\kappa a)^{-1}=1.2$. We also show the results obtained from conventional VH DDLS experiments. Given the difference in initial decay rates, the slower the diffusion of particles, the closer they are to the wall. Further, we show theoretical results using the particle mobility functions in the presence of a wall from Cichocki and Jones [15]. The full lines are predictions, free of adjustable parameters, that include contributions from both translational and rotational diffusive modes. The broken line represents the predicted behavior if translational diffusion was the only means by which the autocorrelation functions decayed. Decay rates

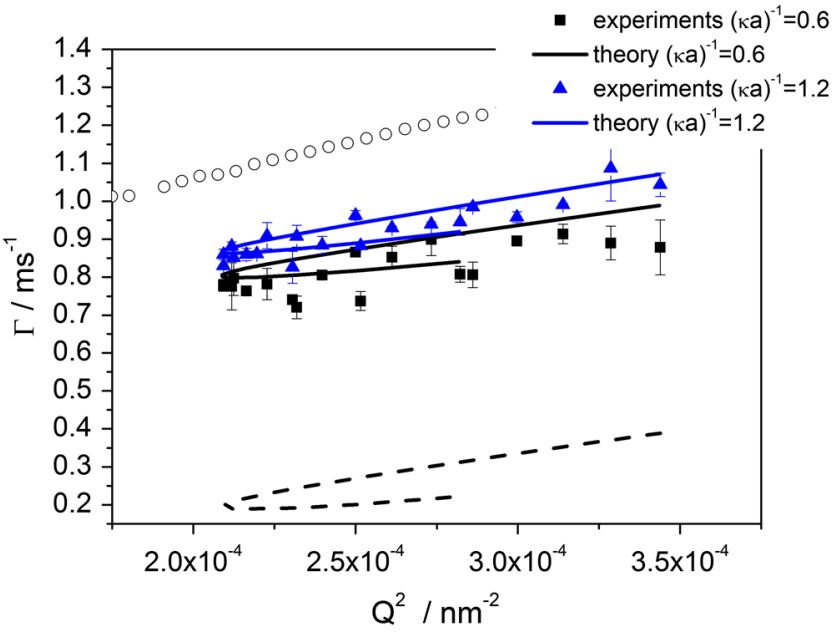

FIG. 2 (color online). Initial relaxation rates as functions of the square of the scattering vector. Open circles: experimental data from a bulk $\mathrm{VH}$ experiment. Black squares or blue triangles: EWDLS results from normalized penetration depths of $(\kappa a)^{-1}=0.6$ and 1.2. Lines (see text). are not strictly functions of $Q^{2}$ alone, as with conventional DDLS experiments, because they also depend on the spatial components of the detector polarizer. Therefore, for a given scattering vector of modulus $Q$, the decay rate changes upon changing the polarization of the detected light. This gives rise to the multivaluedness of the data and the theoretical solid lines in Fig. 2. The specific display of the data in Fig. 2 is chosen for comparison with bulk scattering results. Translational diffusion [Eqs. (4) and (6)] accounts for the $Q^{2}$ dependence of the initial decay $\Gamma$, but is roughly a quarter of the total average diffusion, which also includes rotational modes. We can therefore unambiguously identify the contribution of rotational diffusion. In addition, our experimental methodology confirms the trend toward bulk behavior as the penetration depth is increased.

To resolve the specific contributions from rotation about axes parallel and perpendicular to the wall, the translational augmentation is subtracted from the overall decay rate. It is clear from Eq. (6) that $\Gamma^{\mathrm{VH}}-\Gamma^{t}$ is linear in $n_{S, z}^{2}$, with a positive intercept equal to $2\left\langle D_{\|}^{r}\right\rangle_{\kappa}+4\left\langle D_{\perp}^{r}\right\rangle_{\kappa}$ and a slope of $3\left(\left\langle D_{\|}^{r}\right\rangle_{\kappa}-\left\langle D_{\perp}^{r}\right\rangle_{\kappa}\right)$. While the intercept will always be positive, the sign of the slope depends on whether particles diffuse faster about the axes parallel or perpendicular to the interface. A bulk measurement free of hydrodynamic interactions from walls would yield a slope of zero, as there are no mechanisms by which one particular rotational mode can be slowed down. Subtracting the theoretical expectation for $\Gamma^{t}$ from the experimentally obtained $\Gamma^{\mathrm{VH}}$ for two penetration depths results in the data displayed in Fig. 3.

The negative slopes obtained by linear regression of the data (Fig. 3) confirm the expectation that, due to

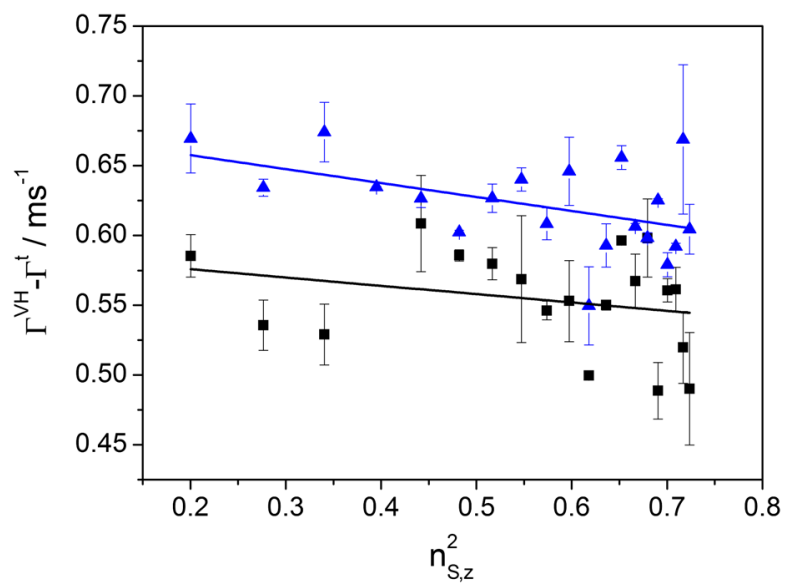

FIG. 3 (color online). Rotational contribution to the initial relaxation rates as a function of the square of the $z$ component of the unit vector $\mathbf{n}_{S}$. Symbols: data obtained from VH experiments at penetration depths of $(\kappa a)^{-1}=0.6$ (black squares) and 1.2 (blue triangles). Full lines: linear regressions representing an attempt to determine the $\kappa$-averaged rotational diffusion coefficients. 


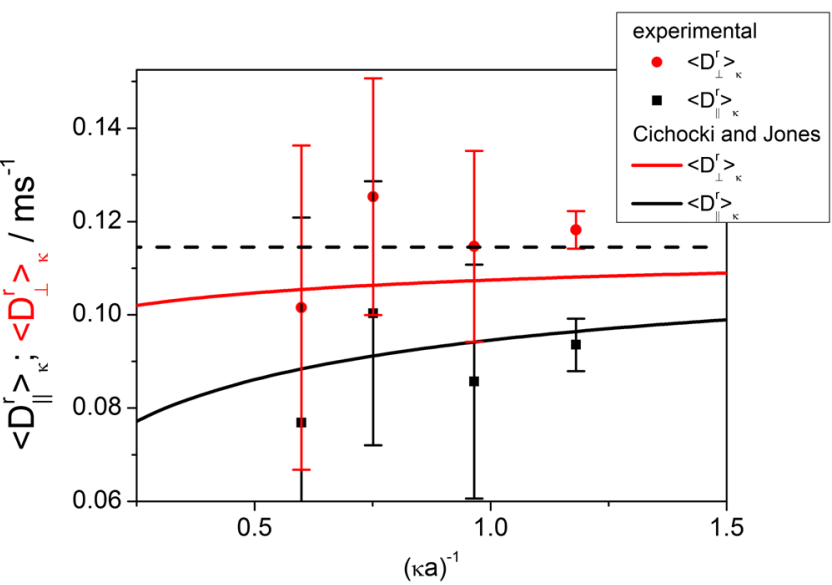

FIG. 4 (color online). $\kappa$-averaged rotational diffusion coefficients as functions of the penetration depth. Symbols and error bars: experimental data as determined by linear regression [Eq. (6)]. Red: rotation around an axis normal to the wall. Black: rotation around an axis lying in the plane of the wall. Lines: see text.

hydrodynamic interactions, $\left\langle D_{\perp}^{r}\right\rangle_{\kappa}>\left\langle D_{\|}^{r}\right\rangle_{\kappa}$, since the parallel rotational particle mobility is more greatly affected by the wall than the perpendicular.

Our ability to extract differences in rotational diffusive rates about the axes parallel and perpendicular to the wall is hampered by experimental uncertainty.

We show in Fig. 4 the $\kappa$-average rotational diffusion about the axes parallel and perpendicular to the wall as a function of penetration depth. In addition, we display the theoretical expectations for each contribution as solid lines. The broken line represents the bulk rotational diffusion constant $D_{0}^{r}$.

Note that the error bars in Fig. 3 are larger than those in Fig. 2. The reason for the significantly larger error bars in Fig. 3 is that the difference $\Gamma^{\mathrm{VH}}-\Gamma^{t}$ is significantly smaller as compared to the values of both $\Gamma^{\mathrm{VH}}$ and $\Gamma^{t}$ themselves. Two relatively large numbers are subtracted to obtain a relatively small number. Moreover, the data in Fig. 4 are obtained from the slopes of the lines in Fig. 3, which amplifies the errors further.

Using a unique experimental setup that allows for scanning over the surface of an entire solid quadrant, we have shown that EWDLS is a reliable tool for investigating nearwall particle dynamics. Experimental values of the initial decay rate of the time-autocorrelation function compare favorably with theoretical predictions free of adjustable parameters, confirming the degree to which hydrodynamic interactions slow down rotational diffusion modes. We are, however, faced with limited precision in determining $\left\langle D_{\| / \perp}^{r}\right\rangle_{\kappa}$. It is conceivable that greater differences to bulk behavior could be obtained by swapping our one-sided geometry for a micro- or nanofluidic channel, where particles feel the presence of multiple walls. The theory for diffusion in such a geometry has been put forward in
Ref. [27]. Rotational motion is pronounced in systems under flow. Depolarized evanescent wave scattering could thus be employed to probe rotational motion near a wall in flowing systems.

The authors acknowledge financial support from the EU through FP7, project Nanodirect (Grant No. NMP4SL-2008-213948). M. L. acknowledges support by the Foundation for Polish Science, which is co-financed by the European Union (EU)-European Regional Development Fund. B. C. acknowledges financial support from the Deutsche Forschungsgemeinschaft Grant No. SFB-TR6, "Physics of Colloidal Dispersions in External Fields," Project A1.

*p.lang@fz-juelich.de

[1] R. J. Hall and D. A. Greenhalgh, Opt. Commun. 40, 417 (1982).

[2] Z. Cheng and T. G. Mason, Phys. Rev. Lett. 90, 018304 (2003).

[3] R. A. Cone, Nat. New Biol. 236, 39 (1972).

[4] R. J. Cherry, A. Bürkli, M. Busslinger, G. Schneider, and G. R. Parish, Nature (London) 263, 389 (1976).

[5] R. A. Alberty and G. G. Hammes, J. Phys. Chem. 62, 154 (1958).

[6] M. Eigen and G. G. Hammes, in Advances in Enzymology and Related Subjects of Biochemistry, edited by F. F. Ford (John Wiley \& Sons, Hoboken, NJ, 1963), Vol. 25, p. 1.

[7] K. Solc and W. H. Stockmayer, J. Chem. Phys. 54, 2981 (1971).

[8] T. M. Squires and S. R. Quake, Rev. Mod. Phys. 77, 977 (2005).

[9] C. L. Rice and R. Whitehead, J. Phys. Chem. 69, 4017 (1965).

[10] D. Psaltis, S. R. Quake, and C. Yang, Nature (London) 442, 381 (2006).

[11] T. Sawetzki, S. Rahmouni, C. Bechinger, and D. W. M. Marr, Proc. Natl. Acad. Sci. U.S.A. 105, 20141 (2008).

[12] C.S. Effenhauser, G. J.M. Bruin, and A. Paulus, Electrophoresis 18, 2203 (1997).

[13] R. H. Carlson, C. V. Gabel, S. S. Chan, and R. H. Austin, Biomed. Microdevices 1, 39 (1998).

[14] J. Happel and H. Brenner, Low Reynolds Number Hydrodynamics (Springer, New York, 1983).

[15] B. Cichocki and R. B. Jones, Physica (Amsterdam) 258A, 273 (1998).

[16] Y. Han, A. Alsayed, M. Nobili, and A. G. Yodh, Phys. Rev. E 80, 011403 (2009).

[17] D. Li, N. Fakhri, M. Pasquali, and S. L. Biswal, Phys. Rev. Lett. 106, 188302 (2011).

[18] S. Lobo, C. Escauriaza, and A. Celedon, Langmuir 27, 2142 (2011).

[19] D. Aarts and R. Dullens, Oxford University (private communication).

[20] K. H. Lan, N. Ostrowsky, and D. Sornette, Phys. Rev. Lett. 57, 17 (1986).

[21] P. Holmqvist, J. K. G. Dhont, and P. R. Lang, Phys. Rev. E 74, 021402 (2006). 
[22] V.N. Michailidou, G. Petekidis, J. W. Swan, and J.F. Brady, Phys. Rev. Lett. 102, 068302 (2009).

[23] B. Loppinet, G. Petekidis, and G. Fytas, Langmuir 14, 4958 (1998).

[24] B. J. Berne and R. Pecora, Dynamic Light Scattering: With Applications to Chemistry, Biology, and Physics (Dover Publications, Mineola, NY, 2000).
[25] See Supplemental Material at http://link.aps.org/ supplemental/10.1103/PhysRevLett.109.098305 for full derivation of the first cumulant.

[26] B. Cichocki, E. Wajnryb, J. Blawzdziewicz, J. K. G. Dhont, and P. R. Lang, J. Chem. Phys. 132, 074704 (2010).

[27] R. B. Jones, J. Chem. Phys. 123, 164705 (2005). 